\title{
Convolutional Neural Network for Brain Tumor Analysis Using MRI Images
}

\author{
Sourabh Hanwat ${ }^{\# 1}$, Chandra J ${ }^{* 2}$ \\ ${ }^{\# 1}$ PG Scholar, Department of Computer Science, \\ CHRIST (Deemed to be University), Bangalore, Karnataka, India \\ ${ }^{\# 2}$ Associate Professor, Department of Computer Science, \\ CHRIST (Deemed to be University), Bangalore, Karnataka, India \\ ${ }^{1}$ sourabh.hanwat@mca.christuniversity.in \\ ${ }^{2}$ chandra.j@christuniversity.in
}

\begin{abstract}
Brain Cancer is one of the most dangerous problems today. Brain Tumor is controlled growth of cancerous or non-cancerous unhealthy cells in the brain. In the present world brain tumor are a very dangerous disease and the main reason for many deaths. Magnetic Resonance Imaging is mostly used the medical image for the brain tumor analysis. The main objective of the paper is to classify the brain tumor various stages using Convolutional Neural Network algorithmbased on Brain MRI images. Brain tumor analysis is done with the help of Convolutional Neural Network and the work is also compared with another popular machine learning classifier like Random Forest and $\mathrm{K}$ Nearest Neighbors. During the comparison, the Convolutional Neural Network is considered as one of the best classifiers for classifying the various stages of a brain tumor. The average accuracy of the brain tumor classification with the help of Convolutional Neural Network classifier is $98 \%$ with cross-entropy is 0.097 and validation accuracy is $\mathbf{7 1 \%}$ so the Convolutional Neural Network is found to be one of the efficient methods for performing different stages of brain tumor classification.
\end{abstract}

Keyword - MRI Image,Convolutional Neural Network, Random forest, K Nearest Neighbors, Median Filter, TensorFlow, Feature Extraction.

\section{INTRODUCTION}

Today we are living in a modern world and observed that the rate of the disease is increasing day by day. The tumor is one of the dangerous diseases which can be on any part of the body which means the irregular shape of the body or lump on body's part. The most dangerous tumor is a brain tumor where the tumor is in the brain and very difficult to cure it. These unhealthy cells can also affect healthy brain cells. It can be classified as a primary and secondary type. A benign tumor is of the primary type which is not cancerous. A malignant tumor is of the second type which stores cancerous cells. A malignant tumor is very dangerous for life. It's very difficult to find a brain tumor and its types.

In the field of medical diagnosis, medical image data is very important. For medical diagnosis, we need to take the medical image information. Mostly used images are CT scan, X-ray, MRI etc. To obtain the internal structure of the brain, Brain Scan medical technique is used. The most used brain scan technique is MRI because of its high resolution. MRI has a lot of information about the brain structure and it also displays any abnormalities within the brain cell [1]. There are many advanced methods in Machine Learning and Deep Learning which are used for image processing. For Classification, we need to use Support Vector Machine (SVM), Neural Network or other related models. There are a lot of learning classifiers available such as ANN, PNN, KNN, C4.5 and Multi-layer Perceptron etc. and each has their own advantages as well as disadvantages. For image classification, Deep Learning Models is widely used for the same reason. Its architecture can represent complex relations. From the past few years, Deep learning has got a lot of attention.

Convolutional Neural Networks (CNN) is a method which used for recognizing the image. It has been competing with other methods established in recent times. CNN's are a collection of neurons with learnable weights and biases.CNN is used for achieve good accuracy in image classification with avoids the preprocessing and It can able to learn complex features automatically from images [2]. TensorFlow library is the best choice for the above requirement which is developed by Google and supports the CNN, RNN and other related neural network models. TensorFlow one of the mostly used library in the field of the image recognition, speech recognition and many other deep learning algorithms. TensorFlow is open source python library which released by Google in 2015 and using it the deep learning models designing, building and training is easy. TensorFlow shows mathematical computation as a graph which has edges and nodes. In the graph edges represent data which is flow one node to another node. 


\section{RELATED WORKS}

Monica Subashini and Sarat Kumar Sahoo [3] hasproposed a method to detect a tumor in brain MRI image. They appliedpulse coupledNeural Network for enhancing the brain MRI images and back propagation network for classification the brain MRI images. They observe that image enhancement and segmentation using pulsecoupled Neural Networkand back propagation network helps in detection of brain tumor in MRI image.

Danda Shashank Reddy et al., [4] has used Naïve Bayes and decision tree algorithms for prediction the brain tumor based on the tumor Origination, symptoms, treatment and Tumor Occurrence. Based on the result they observed that decision tree is simple and easy to predict the brain tumor treatment than the Naïve Bayes algorithm.

Nikita Singh and Naveen Choudhary [5] has proposed a new approach to classifying the brain tumor MRIimageusing Hellinger decision method HD-Tree and HD-Forest algorithms. In the proposed system they have used 97 brain tumor MRI images to classify based on the different features like symptoms of brain tumor, centroid and shapes. The performance of HD-forest was better than LA-SVM with 96.50\% where LA-SVM accuracy was $96 \%$.

A. Sankari and S. Vigneshwari [6] has proposed a CNN based brain tumor classification method. The proposed work used the non-linearity activation function which is a leaky rectifier linear unit (LReLU). They mainly focus on Basic Feature which is Entropy, Mean and Standard deviation of the images. they analysed that CNN is working better for representing the complex features of brain tumor tissues.

Yuehao Pan et al., [7] has used brain tumor MRI images for getting useful information for classifying brain tumor grading. In the proposed method they used CNN algorithms for developed a brain tumor grading system. The performance of CNN based on sensitivity and specificity is improved by $18 \%$ compared to the Artificial Neural Network.

G Vijay Kumar and GV Raju [8] has proposedArtificial neural network base early brain cancer detection system. Proposed system used neuro fuzzy logic for Brain tumor detection. They observed that the proposed model detection time and accuracy is 50-60 \% improved compared to the existing neuro classifier.

Dena Nadir George et al., [9] showed an accuracy of Multi-layer Perceptron is better than C4.5 classifier. The proposed approach used C4.5 and MLP based on the major axis length, minor axis length, Euler Number feature shape of tumor. Proposed approach trained with 174 images of brain tumor MRI. The Multi-layer Perceptron classification rate was $95.2 \%$ and C4.5 classification rate was $91.1 \%$.

Eman Ali et al., [10] has proposed a neuro fuzzy approach for classification of brain tumors MRI images based on the tumor shape and size. The proposed system used different classification algorithms with k-means and CBIR segmentation methods. They observedthat the performance of Tree Augmented Naive Bayes Nearest Neighbor (TANNN) algorithm is better than other algorithms and the classification time rate of K-Nearest Neighbor is minimum compared to other classification algorithms.

Sunil L. Bangare et al., [11] used Support Vector Machine classifier for brain tumor tissues. They proposed an effective method approach for classification brain tumor with Genetic algorithm and SVM classifier.The Genetic algorithm used for feature extraction and SVM for classification. The proposed system detects the tumor based onmean, mod, and median values of the tumor region and classify the MRI brain tumor type.

Vaishnavi S. Mehekare et al., [12] has proposed a system for detection of brain tumor using CNN classifier and Local Binary Patterns (LBP) feature extracting method. Relu Activation function is used in the CNN algorithm. The proposed method was tested using 100 images and it provides $86 \%$ accuracy.

J. Seetha and S. Selvakumar Raja [13] has proposed an automatic brain tumor detection model using the CNN classification algorithmfor the classification of tumor or non-tumor detection. The classification achieves $97.5 \%$ accuracy on CNN which is better than SVM and DNN classification algorithms.

According to related work, it is observed that theConvolutional Neural Network is considered as one of the bestalgorithms for brain tumor MRI image classification.

\section{Methodology}

In this section, the brain tumor MRI image classification algorithm are explained based on the Convolutional Neural Network, Random forest and K Nearest Neighbors classification techniques. Brain tumor classification is very important for medical diagnosis and high accuracy is also needed when human life is involved.The proposed methodconsists ofsixdifferent steps involved for the classification of brain tumor MRI image which is shown in figure 1. It involved input image, image pre-processing, image segmentation, feature extraction, classification and performance evaluation of classifiers. 


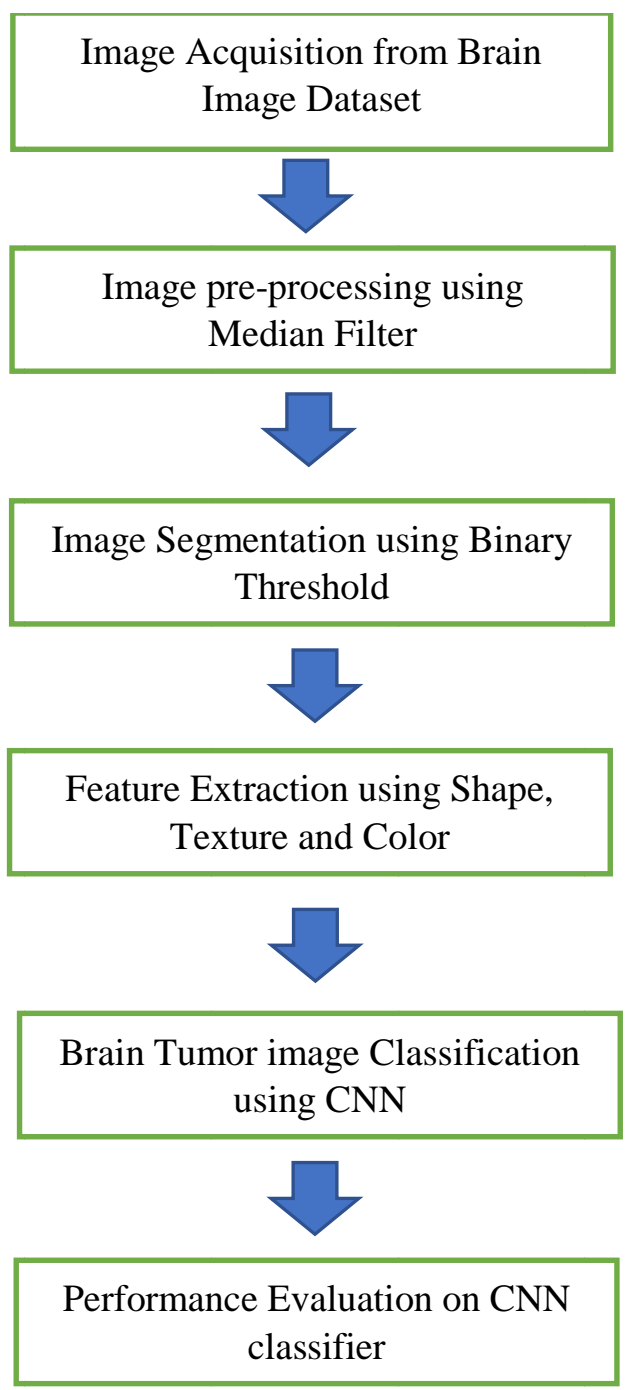

Fig. 1. Proposed Framework for Brain Tumor Classification

The proposed framework begins with loading the input MRI image from the dataset, image pre-processing used dilate function for skull removal and median filtering for removing the image noises of the brain tumor image.The brain MRI image segmentation is implemented with the help of binary threshold method with erosion and dilation operation based on morphological functions. The various featureextracted using shape, texture and colors based on brain tumor MRI image. After the feature extraction MRI brain tumor classification is done with the help of Convolutional Neural Network and the classification accuracy is compared against Random forest and K Nearest Neighbors classifiers.

\section{A. Image Acquisition}

The Brain MRI image dataset has downloaded from the Kaggle. MRI dataset has 94 brain MRI images including benign, malignant and normal. MRI brain images were taken as input to pre-processing step.

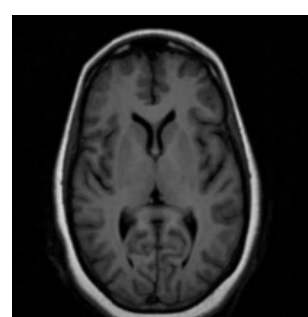

Brain MRI without tumor

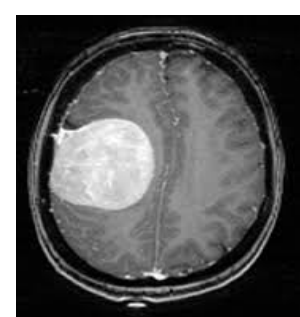

Brain MRI with tumor

Fig. 2. Brain MRI images 
The appearance of the brain MRI image has generated with the help of MRI scanner. the strong magnetic fields and radio waves help to generate MRI image. MRI directly effects the treatment of patients. The tumor has highlighted in the MRI image with white color.

\section{B. Pre-Processing}

The pre-processing is a very important step in image processing for improving the quality of the image. Removing noise, resize image is the basic step in the image pre-processing. The first step of the pre-processing is converting MRI images into the Grayscale image. Skull masking and the image noise removing is a very important step in brain MRI image classification it will help to improve classification accuracy rate. Skull Masking is the process of removing brain tissues from non-brain tissue from the brain [16]. It improves the accuracy of diagnosis and also helps in improving the classification result. In this research work imclearborder, Bwareafilt and imdilate method are used for skull masking.

\section{Segmentation}

In the Segmentation technique, separate an image into multiple slices and object regions [17]. The major advantage of using Image Segmentation is a way to change the image into easier recognizable and analysable format. The Binary thresholding techniques are used in this proposed work. The MRI images have numbers of pixel intensity, which is greater than or less than to threshold value.

$$
\operatorname{dat}(x, y)=\left\{\begin{array}{c}
\text { maxValifsrc }(x, y)>\text { thresh } \\
\text { 0otherwise }
\end{array}\right.
$$

The Threshold equation (1) takes the $\mathrm{x}$ and $\mathrm{y}$ values as an input which is basically the pixel values of images with respect to $\mathrm{x}$-axis and y-axis. "MaxVal" is the set threshold value which compares with input values, when the input is greater than the set threshold value it gives output is set Maxval value and it is shown with white color in gray images. when the input pixel intensity values are less than the set threshold, its output is black color (assign 0 value). The Morphological techniques also used in this proposed work with segmentation techniques. The morphological action is normally performed on binary images. It processes the operations based on shape and it has a wide set of the image processing operation. Erosion and Dilation are two methods of morphological operations which used in this proposed work.

In this proposed work Erosion and dilation operations used together. Two main steps of the erosion and dilation morphological operation are Opening and closing. The first step is the opening of the MRI binary image. The main work of opening operation is open up a gap which is present in between object and connect that to a small collection of pixels. After setting of the bridge, the erosion again restored with their actual size using dilation. If the binary image has been opened then the subsequent opened same structured elements have not affected on that image. After completing the opening operations next step is the closing operation. Based on the Closing operation while keeping the original region sizes, the erosion and dilation can handle different hole in the image region.

\section{Feature Extraction}

Feature extraction method used to get the very important features in the images for reducing the process time and complexity in the image classification [18]. Global features are extracted from the image, from all areas in the input images. Global features of the MRI image can be extracted by the Haralick, Hu Moment, color Histogram, Hough transform, Discreate Cosine Transform (DCT), Discrete Random Transform (DRT) etc. current research work used Hu Moment, Haralick and color Histogram for extractingvarious features like shape, texture and color.

Gray-level co-occurrence matrix (GLCM)proposed by Haralick whichis one of the most famous and widely used texture measures methods. Haralick proposed fourteen measures of textural features. GLCM is able to measures many features from the input image like Contrast, Homogeneity, Entropy, Correlation etc. The Contrast is used for measuring the differences of intensity level between the given pixel and its connected pixel. The contrast level is high means intensity differences in GLCM is alsohigh. The Entropy is basically the randomly arranges of not in ordered values of MRI image. If the value of entropy is biggest, the co-occurrence matrix's all elements are in small and same, and also the value of elementsare equal.

The Histogram and Hu moment used for features extraction. They extract the global features from the MRI brain images. The Histogram used statistic information about gray levels of the image. Hu Moment used to define, characterize and quantify the shape of an object in the image. Its normally extracted from the outline of the object in the image. Hu moment also able to extract image shape feature vector to represent the shape of the object. 


\section{E. Classification}

Classification is the process of classifying the items according to its type and pattern and selecting the suitable classifier results in accuracy and improved performance for various datasets [19].

1) ConvolutionNeural Network Classifier: The ConvolutionNeural Network (CNN) classifier is usedmainly for image and video recognition.The CNN is able for automatically learning the respective feature for data itself. The CNN follows few steps like receiving different inputs, calculating the sum of their weights, forward output to activation function and respond with the desired output. Based on CNN classification, the Brain MRI images important features like lines, edges, and object etc. complex features automatically able to identify withmore accurately.

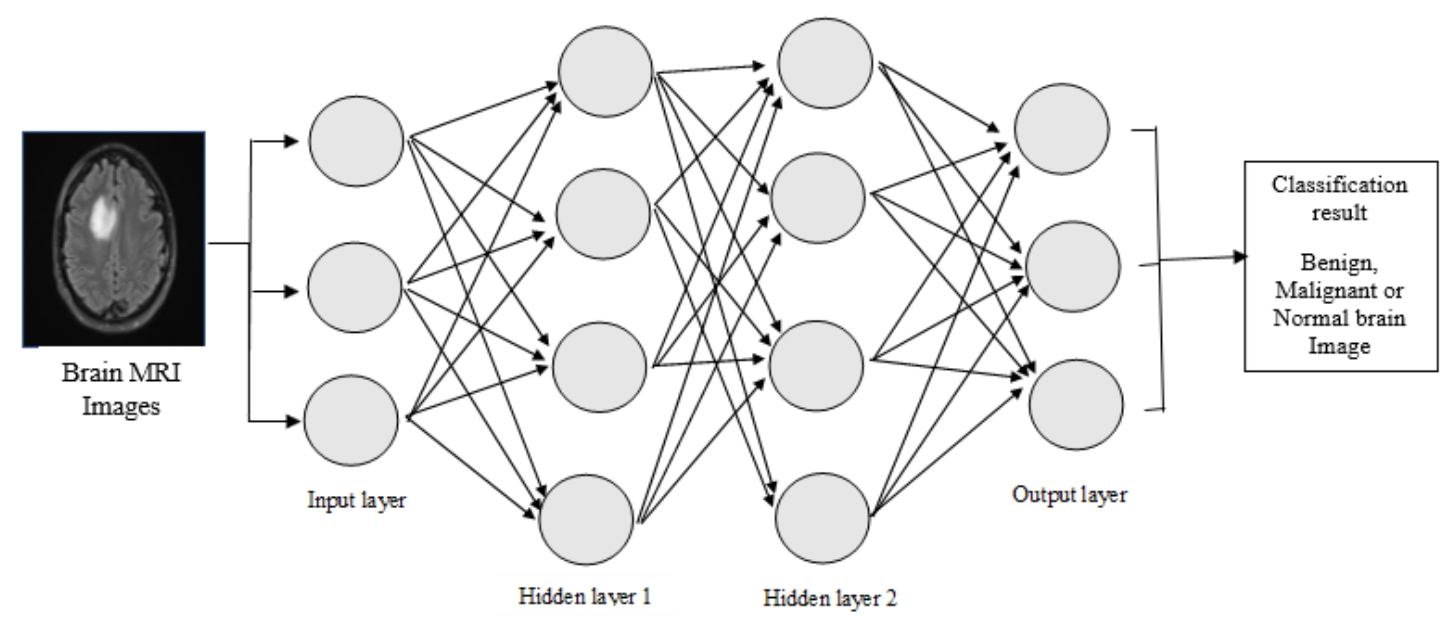

Fig. 3. Structure of a Convolution Neural Network

The Structure of the Convolution Neural Networkis shown in Fig. 3. The Convolution Neural Network has majorly 4 layers. Input layer, Convolution layer, pooling layer and Fully connected layer [20]. The equation (2) shown the Rectified Linear Unit (ReLU) activation function which has input $\mathrm{x}$ as an input value. If the input value is less than 0 , in this time the output is 0 . If the input value is greater than 0 , in this time the output is not changed the same equal to the input.

$$
\operatorname{ReLU}(x)=\left\{\begin{array}{l}
0 \text { if } x<0 \\
x \text { if } x \geq 0
\end{array}\right.
$$

The Convolution Neural Network is built with the help of TensorFlow in python. TensorFlowis python based opensource library. It helps us for numerical computation in time of CNN classification model creation. It can train and run deep neural networks for classification models for the neural network. TensorFlow can support production predication scale, with the same models used for training. It provides Activation Functions and applying dropout regularization. Inception-v3 is the pre-trained models on the TensorFlow and it is a mostly used image recognition model. The Inception-v3 model has convolutions, pooling, max pooling, and fully connected layers building blocks.

2) Random Forest Classifier:The Random Forest is a technique to get large groups of binary decision trees. It is mainly based on two random processes. Randomly sampled training set. Another one is introduced in the building process of the tree [20]. For obtaining bootstrap set need to use the first one. In each node, only select features are used to search for the best split. A simple flow of the random forestis shown in the fig. 4, which is shown that all the random forest has its own result and based on the numbers of result classifier chosen the correct result. 


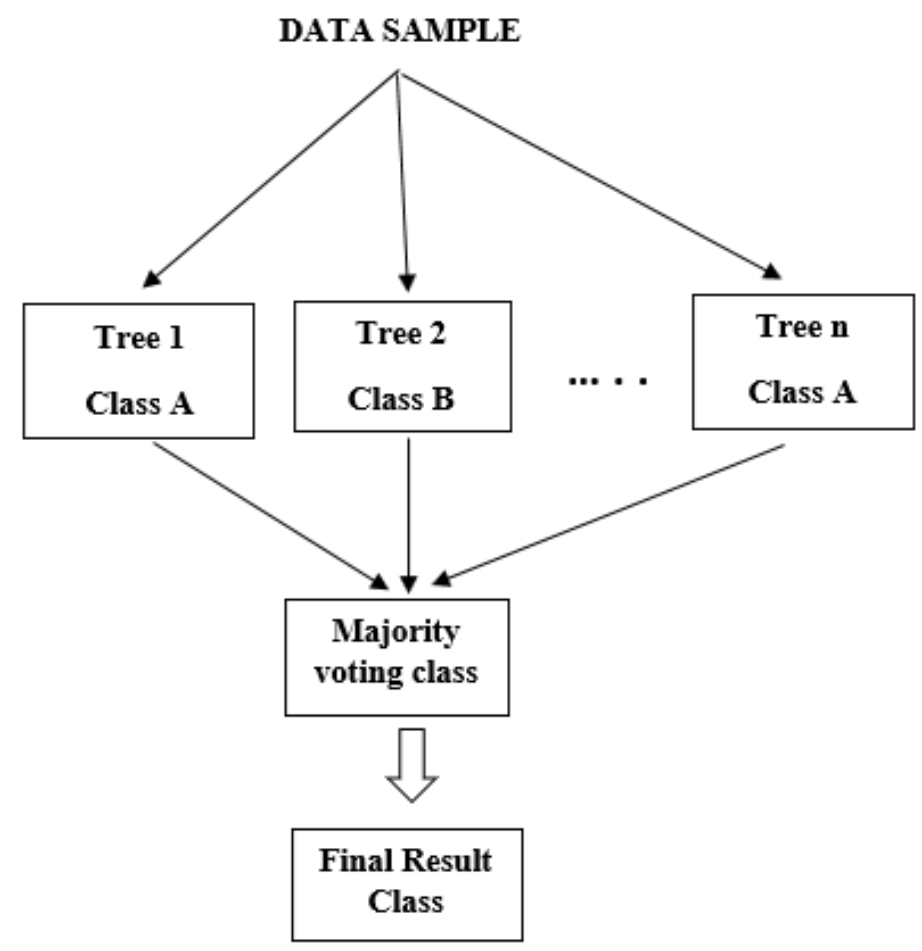

Fig. 4. Random Forest Workflow

Random forest is one of the popular supervised learning algorithms which is most flexible and easy to use. It has many decision trees that why it is the most accurate and robust method. In this proposed work, Scikit-learn library used for importing the Random forest classifier. The extracted features from brain MRI is used for classifying the brain tumor MRI. Random forest creates a group of many decision trees and each decision tree give one result. The final random forest classification result depends on the aggregates the votes from different decision tree.

3) K-Nearest Neighbors Classifier:K Nearest Neighbors classifier is Supervised classification method. During the training time, without having any previous information about the structure of the dataset it classifies the training set based on the $\mathrm{k}$ value. In the proposed work Scikit-learn python library used for importing the KNN classifier in the program. K Nearest Neighbors algorithm known as a lazy learner as well because it doesn't learn from the training data but memorize the training dataset. The algorithm is based on similar features. As there is no training phase, $\mathrm{K}$ Nearest Neighbors keeps all training data and used it during the testing phase. How closely our features resemble out training set determines how images classify a given data point. The outcome of this algorithm is a discrete value which is based on the majority of votes from its neighsbors. If the $\mathrm{k}$ value rises the confidence in prediction improves [21].

K Nearest Neighborsclassify the input images based on the value of its neighbors and value of $k$. All the distance measures are only for continuous variables. If a value of $k$ value is big than the overall noise reduces. The final classification is decided based on the result of the minimum distance. Minimum distance means the value or features is more suitable for the closest group. KNN is work better when the input dataset is continuous but it also works well for another type of datasets.

\section{RESUlts AND Discussions}

This section explains the result and discussions about the proposed methodology. Its start with dataset description then image pre-processing where three types of noise removing method is compared and analyses the best noise removing method. The CNN classifier is used for classification and compared with another image classifier.

\section{A. Dataset Description}

The MRI images are mostly used images in the medical. A data set of 94 MRI images has been taken for this research work. In the data set abnormal images have been divided into two categories benign and malignant. In this research work, images are classified into three types of Brain MRI which are Benign, Malignant and Normal Brain. 


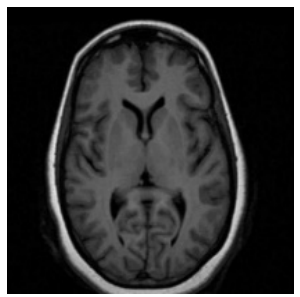

Normal MRI

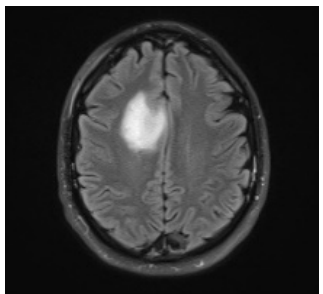

Benign Tumor

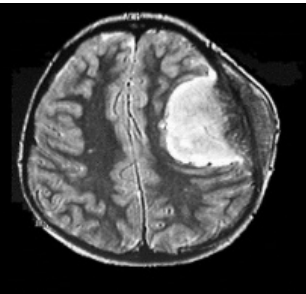

Malignant Tumor

Fig. 5. Dataset MRI images

Fig. 5 shown the MRI images of the normal MRI, benign and malignant tumor MRI. The normal MRI has no abnormal tissue but Benign and Malignant MRI has abnormal tissues which highlighted with white color inMRI image. Benign has fix edges but malignant has not fix edges.

\section{B. Pre-processing}

The actions involved in Skull masking is an imclearborder function used for removing the background and bwareafit function for extract the skull and brain. At last imdilate function is used for mask out the brain.

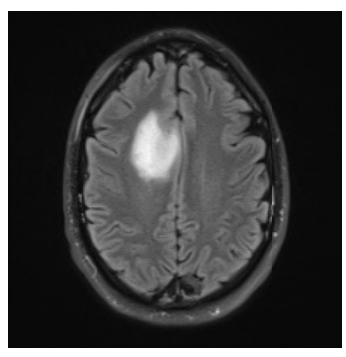

(A)

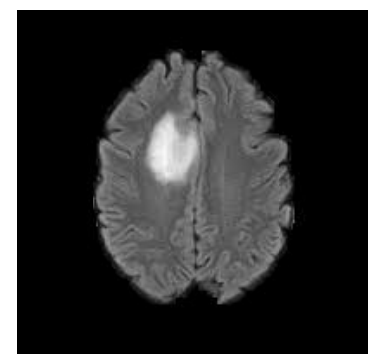

(B)

MRI image with a skull

MRI image without skull

Fig. 6. Skull Masking

Fig. 6 shows Skull masking on MRI image where after applying the skull masking brain MRI is one has brain tissues. The input image 6(A) has skull after applying the skull masking the output image 6(B) has not skull and the brain shape is clear.

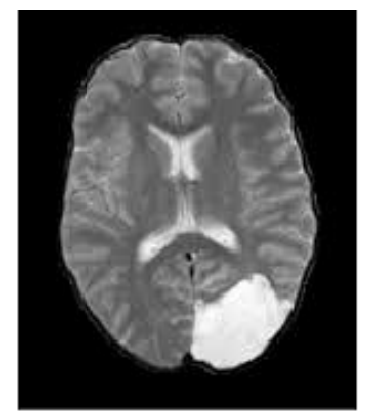

(A) Input MRI image

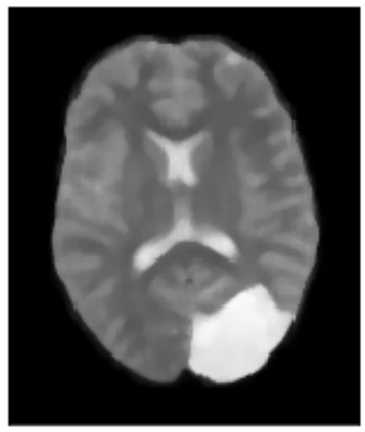

(B) Median filter

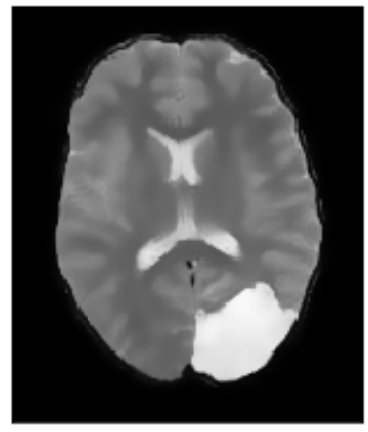

(C) Bilateral filter

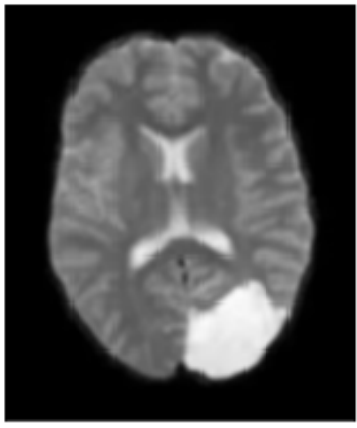

(D) Gaussian filter

Fig. 7. Noise removing using Median, Bilateral and Gaussian filter with input and outputresult MRI image 
Fig.7 shows different noise removing methods with input and result image. Median filter, Gaussian filter, and the Bilateral filter output result shown in 7(B), 7(C) and 7(D). The median filter is a digital filtering technique. In image processing, Median filtering work for image noise removing and its calculated by ordering all the pixel values from the image into numerical order, then middle pixel value will be replacing all the pixel value from the image [18]. In the Gaussian filter, the Gaussian kernel will also be used. Only the kernel width and height will be specified as a positive an odd number. The Gaussian filter reduces noise in the image [21]. The bilateral filter used when the image has edges sharp. It does not take pixels which has the same intensity and blurs the edges.

All noise removing techniques are useful at removing noise in smooth patches or regions. When the images have edges or small level of noise, the median filter is performed better than others noise removing techniques. The median filter, work is that the inaccurate pixel can often be brought back as an average value of neighboring pixels.

\section{Segmentation}

Images have pixels and all pixels are having some values and based on the threshold level, which is lower than a threshold or greater than the threshold value in the binary threshold, the higher pixels values for foreground and assign one value 255 (white color). Rest pixel values as background with 0 value (black color).

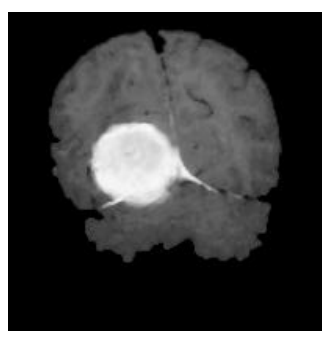

Without segmentation MRI image

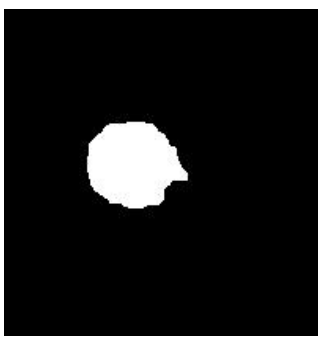

MRI image After Segmentation and Morphology

Fig. 8: Segmentation and Morphology

Fig. 8 shown threshold segmentation with morphological operations. The morphological operation Erosion skips the boundaries of the center object and used to moderate the features of an image. Dilation helps for an increase in the object area and used to highlight features.

\section{Classification}

The CNN, K Nearest Neighbors and Random Forest classifier for testing after successfully training images. The CNN is taking images directly for training the model and KNN as well as Random forest are taking the features of the dataset for training the model for brain tumor classification.

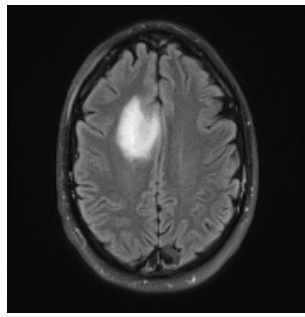

(A) Original Benign MRI

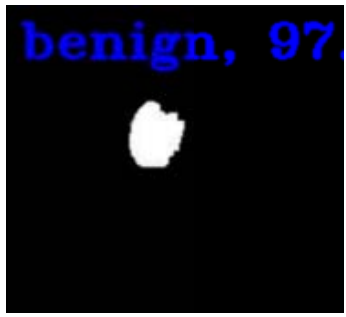

(B) Result Output MRI Image with 97\% Benign Tumor

Fig. 9: CNN classification result for Benign MRI image

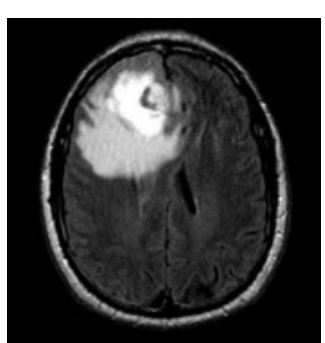

(A) Original Malignant MRI

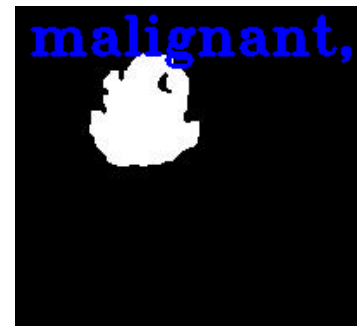

(B) Result Output MRI Image with 98\% Benign Tumor

Fig. 10: CNN classification Result with Malignant MRI image 
In fig. 9 shown that CNN classification result forBenign MRI images with good accuracy rate. In the fig. 9 The accuracy achieved for Begin images is $97 \%$ and fig. 10 shown that Malignant image got $98 \%$ accuracy. The class label is written on the top of the image. Test image classification result shows in the resulting image.

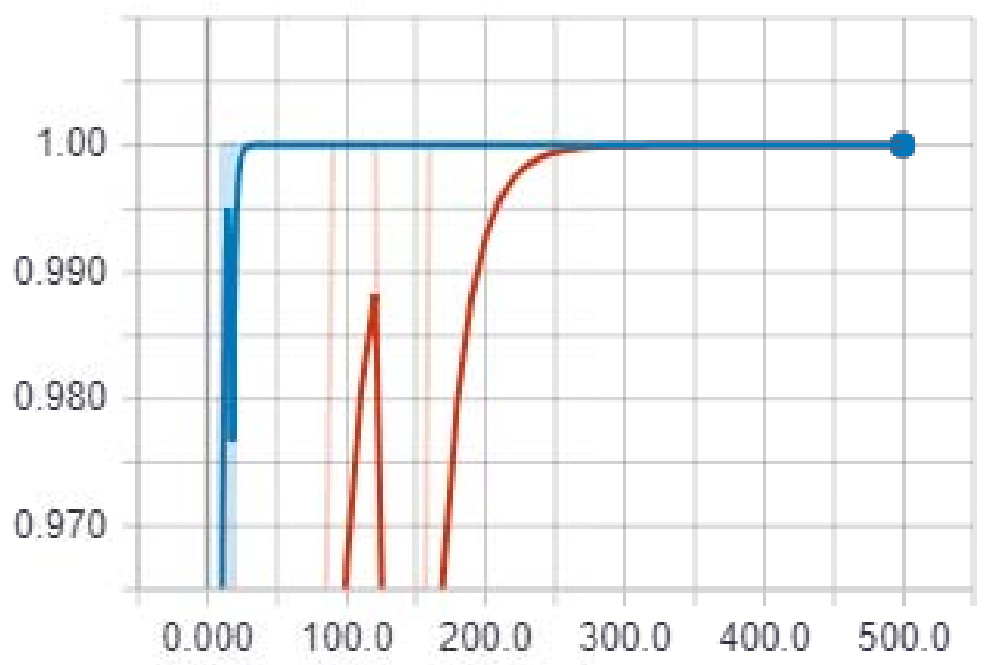

Fig.11. Performance of Accuracy using training and validation

In Fig11 shows the difference of accuracy based on the brain tumor dataset which used for training the model. The two lines used in this graph first one, Orange line represents the training, and second is the green line represents the validation. Validation accuracy basically is the precision. In the figure $\mathrm{X}$ axis is steps of model and $\mathrm{Y}$ axis is values of model accuracy.

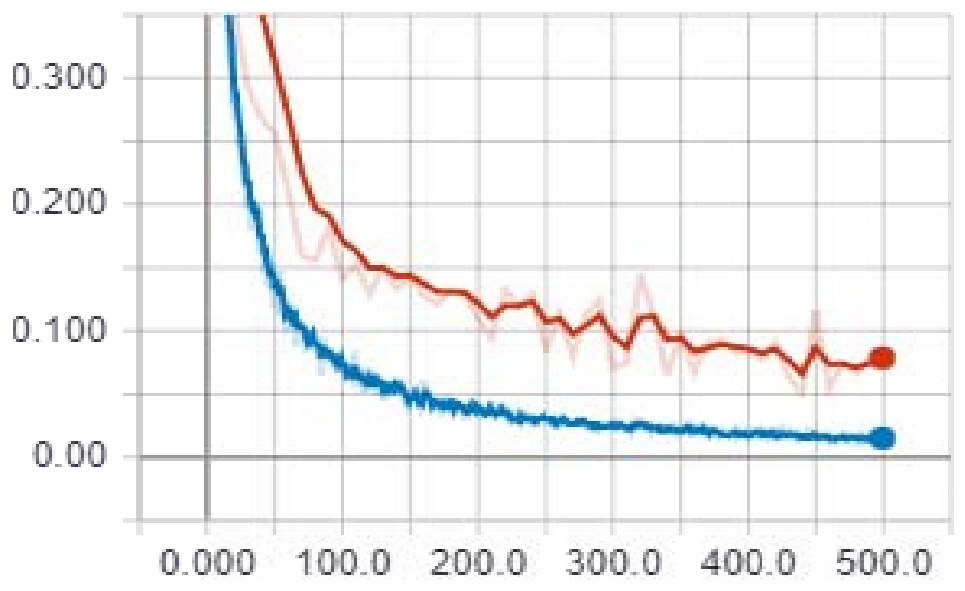

Fig12.Performance of cross entropy for training and validation

In Fig.12 shows that the measure of error between calculated outputs and the wanted goal outputs of the training data. Cross-entropy is basically a loss function. Cross-entropy gives information about the process and moving of process. The minimum number of are better in the cross entropy.In this figure $\mathrm{X}$ axis is Steps and $\mathrm{Y}$ axis is values of model Cross-entropy.

The Random Forest classifier given the average classification result. For training its take 73 training data and 15 testing data. The classification result of random forests is depending on the decision trees and their results.The RF algorithm has the overall classification result with $89 \%$.

The KNN classifier takes 73 brain MRI images training data and 15 brain MRI images testing data. The features of image dataset are mainly responsible for classification result. All the features are used for training the model for KNN. The KNN algorithm has the overall classification result is $88 \%$. The classification accuracy of KNN classifier depends on the different $k$ nearest samples [21]. 
TABLE 1. PERFORMANCE EVALUATION OF ALGORITHMS

\begin{tabular}{|c|c|c|c|}
\hline S. No. & Algorithms & Dataset Size & Classification Rate (\%) \\
\hline 1 & CNN & 94 & $98 \%$ \\
\hline 2 & RF & 88 & $89 \%$ \\
\hline 3 & KNN & 88 & $88 \%$ \\
\hline
\end{tabular}

The Table 1represent the performance of variousclassifier. CNN classifier performs is good compared to RF and KNN with the used MRI image dataset. The accuracy of the brain tumor MRI images using CNN was around $98 \%$ while the accuracy of RF was $89 \%$. CNN is a deep learning algorithm and this table shown that deep learning is good for image type dataset. In the CNN the features are automatically selected by the CNN.

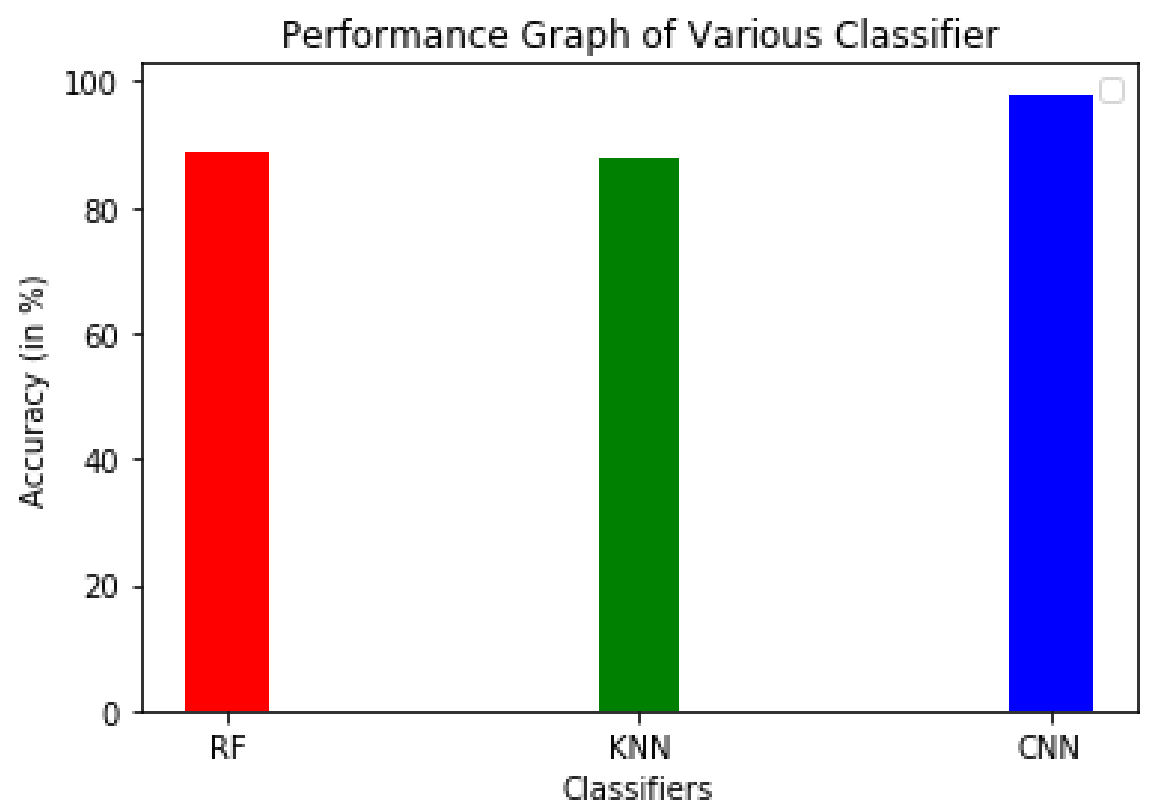

Fig13. Performance Graph on Classifiers Accuracy

After completing the classification performance evaluation is shown in fig. 13. The accuracy results are shown as a graphical form with like a blue point. Fig.13 shows that CNN classifier is work better compare to KNN and RF.

In this research work, the CNN algorithm used for classifying a brain tumor with the help of the MRI images. The accuracy for the training of the model is 98\% with cross-entropy is 0.097 and validation accuracy is $71 \%$ which is better than the result of the Random Forest and k-Nearest Neighbors classifier.

\section{CONCLUSION}

In this research work, three different classification algorithms used for brain tumor classification as a benign, malignant and normal MRI images. Proposed method used Dilate and Bwareafilt method for skull removing. The median filter used for removing noise of the image. Binary threshold with morphological segmentation helped for highlighting the tumor in the MRI images. In the proposed method Hu moment, Haralick and Histogram helped to extract the global features. The classification performed with the help of CNN, RF, and KNN. The CNN is achieved maximum accuracy of $98 \%$ with cross-entropy is 0.097 and validation accuracy is $71 \%$. The Random Forests achieved $80 \%$ of accuracy and K-Nearest Neighbors achieved $74 \%$ of accuracy which is lesser than CNN. The analysis of research work, results proved that Convolutional Neural Network image classification method is better compares to other machine leaning classification methods.

\section{ACKNOWLEDGMENT}

we would like to thank Prof. Joy Paulose, for his valuable suggestions and guidance for completing this research work. we must admit that he always inspires and motivated us for this work. 


\section{REFERENCES}

[1] Heba Mohsen, El-Sayed A., El-Dahshan, El-Sayed M. El-Horbaty, Abdel-Badeeh M. Salem, "Classification using deep learning neural networks for brain tumors”, Future Computing and Informatics Journal, ScienceDirect 2018.

[2] Wang Mengqiao,Yang Jie, Chen Yilei, Wang Hao, "The Multimodal Brain Tumor Image Segmentation Based on Convolutional

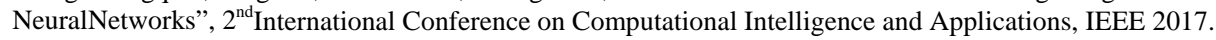

[3] Monica Subashini.M, Sarat Kumar Sahoo,“Brain MR Image Segmentation for TumorDetection using Artificial Neural Networks”, International Journal of Engineering and Technology (IJET), Vol.5, No 2,Apr-May 2013.

[4] Danda Shashank Reddy, Chinta Naga Harshitha, Carmel Mary Belinda,” Brain Tumor prediction using naïve Bayes classifier and decision tree algorithms", International Journal of Engineering and Technology, 2018.

[5] Nikita Singh, Naveen Choudhary, "Classification of brain tumor types using multiclass kernel-based Hellinger decision method for HD-tree and HD-forest”, International Journal of Engineering and Technology, 2018.

[6] A. Sankari, S. Vigneshwari.” Automatic Tumor Segmentation Using Convolutional Neural Networks”, Third International Conference on Science Technology Engineering and Management (ICONSTEM), 2017.

[7] Yuehao Pan, Weimin Huang, Zhiping Lin, Wanzheng Zhu, Jiayin Zhou, Jocelyn Wong, Zhongxiang Ding,” Brain Tumor Grading Based on Neural Networks and Convolutional Neural Networks”, IEEE, 2015.

[8] G Vijay Kumar, GV Raju, "Biological Early Brain CancerDetection Using Artificial NeuralNetwork", International Journal on Computer Science and Engineering, Vol. 02, 2010.

[9] Dena Nadir George, Hashem B. Jehlol, AnwerSubhi Abdul Hussein Oleiwi,” Brain Tumor Detection Using Shape Features and Machine Learning Algorithms”, International Journal of Scientific \& Engineering Research, volume 6, issue 12, December-2015.

[10] Eman M. Ali, Ahmed F. Seddik, Mohamed H. Haggag," Using Data Mining Techniques for Children Brain Tumors Classification Based on Magnetic Resonance Imaging”, International Journal of Computer Applications, 2015.

[11] Sunil L. Bangare, G. Pradeepini, Shrishailappa T. Patil,” Brain Tumor Classification Using Mixed Method Approach”, International Conference on Information, Communication and Embedded Systems (ICICES) 2017.

[12] Vaishnavi S. Mehekare, S.R.Ganorkar"Brain Tumor Detection Using Neural Network”, International Journal of Advanced Research in Electrical, Electronics and Instrumentation Engineering, Vol. 6, Issue 5, May 2017.

[13] J. Seetha, S. Selvakumar Raja, "Brain Tumor Classification Using Convolutional Neural Networks", Biomedical \& Pharmacology Journal, Vol.11, Sep 2018.

[14] Wang Mengqiao, Yang Jie, Chen Yilei, Wang HaO,” The Multimodal Brain Tumor Image Segmentation Based on Convolutional Neural Networks", $2^{\text {nd }}$ IEEE International Conference on Computational Intelligence and Applications, 2017.

[15] V. Amsaveni, N. Albert Singh, "Detection of Brain Tumor using Neural Network", IEEE $4^{\text {th }}$ ICCCNT,2003.

[16] Bhavana Ghotckar, K. J. Mahajan," Brain tumor Detection and Classification SVM”, National Conference on Innovative Trends in Science and Engineering, 2016.

[17] Aparna M. Nichat, S. A. Ladhake,” Brain Tumor Segmentation and Classification Using Modified FCM and SVM Classifier”, International Journal of Advanced Research in Computer and Communication Engineering, Vol.5, Issue 4, April 2016.

[18] Hifzan Ahmed, Shailja Shukla, "Comparative Analysis of Global Feature Extraction Methods for Off-line Signature Recognition”, International Journal of Computer Applications, Volume 48-No.23, June 2012.

[19] Kailash D. Kharat, Pradyumna P. Kulkarni, M.B. Nagori,” Brain Tumor Classification Using Neural Network-Based Methods”, International Journal of Computer Science and Informatics, 2014.

[20] Laszlo Lefkovits, SzidoniaLeidonia, Mircea-Florin Vaida, "Brain Tumor Segmentation Based on Random Forest”, Memories of the Scientific Sections of the Romanian Academy,2016.

[21] Nikita V. Chavan, B.D. Jadhav, P.M. Patil, "Detection and Classification of Brain Tumors", International Journal of Computer Applications, Vol. 112, Feb 2015.

\section{AUTHOR PROFILE}

Sourabh Hanwat is PG Scholarin Department of Computer Science, CHRIST (Deemed to be University), Bangalore, Karnataka, India. He has studied his B.Sc. in information Technology from Makhanlal Chaturvedi RashtriyaPatrakaritaAvam Sanchar Vishwavidyalaya (2013 - 2016), Bhopal (Madhya Pradesh). Hisresearch area is Machine learning and Deep learning.

Chandra J. is an Associate Professor at Department of Computer Science, CHRIST (Deemed to be University), Bangalore, Karnataka, India. She holds a Masters in Computer Applications from Bharathidasan University. She has MPhil in Computer Science from Vinayaka Mission University and PhD in Hindustan University. Her research interests include Artificial Neural Network, Data Mining, Genetic algorithm, genomics, Machine Learning, big data analytics and Predictive analytics. 